\title{
Upper Respiratory Tract Infections
}

\author{
Neemisha Jain, R. Lodha and S.K. Kabra \\ Department of Pediatrics, Division of Pediatric Pulmonology, All India Institute of Medical Sciences, \\ New Delhi, India.
}

\begin{abstract}
Acute respiratory infections accounts for $20-40 \%$ of outpatient and $12-35 \%$ of inpatient attendance in a general hospital. Upper respiratory tract infections including nasopharyngitis, pharyngitis, tonsillitis and otitis media constitute $87.5 \%$ of the total episodes of respiratory infections. The vast majority of acute upper respiratory tract infections are caused by viruses. Common cold is caused by viruses in most circumstances and does not require antimicrobial agent unless it is complicated by acute otitis media with effusion, tonsillitis, sinusitis, and lower respiratory tract infection. Sinusitis is commonly associated with common cold. Most instances of rhinosinusitis are viral and therefore, resolve spontaneously without antimicrobial therapy. The most common bacterial agents causing sinusitis are S. pneumoniae, H. influenzae, M. catarrhalis, $S$. aureus and S. pyogenes. Amoxycillin is antibacterial of choice. The alternative drugs are cefaclor or cephalexin. The latter becomes first line if sinusitis is recurrent or chronic. Acute pharyngitis is commonly caused by viruses and does not need antibiotics. About $15 \%$ of the episodes may be due to Group A beta hemolytic streptococcus (GABS). Early initiation of antibiotics in pharyngitis due to GABS can prevent complications such as acute rheumatic fever. The drug of choice is penicillin for 10-14 days. The alternative medications include oral cephalosporins (cefaclor, cephalexin), amoxicillin or macrolides. [Indian J Pediatr 2001; 68 (12) : 1135-1138]
\end{abstract}

Key words : Upper respiratory tract infections; Pharyngitis; Sinusitis; Nasopharyngitis

Acute respiratory infections are a major cause of morbidity and mortality in children and of particular significance in developing countries like India. Outpatient attendance attributed to acute respiratory infections is as high as $20-40 \%$ of all outpatients and $12-35 \%$ of in patients. ${ }^{1}$ In a study in rural zone of Delhi with an estimated population of 3700 , the prevalence of acute respiratory infection was $12.1 \%$ for the under-fives as a whole. ${ }^{2}$ The overall incidence of acute respiratory infection in the under-fives was observed to be 2.5 episodes per child per year, of which, 2.2 episodes per year per child were upper respiratory tract infections. Upper respiratory tract infections including nasopharyngitis, pharyngitis, tonsillitis and otitis media constituted $87.5 \%$ of the total episodes of infection. Lower respiratory tract infections including pneumonia and bronchiolitis constituted the remaining $12.5 \%{ }^{2}$

In a survey of acute respiratory infection in a rural area of South India, the prevalence of acute respiratory infection was $7.6 \%$ in a total of 10,951 children below 5 years. Majority of acute respiratory infection was mild (defined by cough + respiratory rate $<50$ ) only $1.7 \%$ episodes were severe in nature (cough + respiratory rate $>50+$ chest indrawing/inability to drink). ${ }^{3}$

Thus, acute respiratory infection constitutes a significant problem in childhood and the majority of these infections are upper respiratory infection. Bacterial

Reprint requests : Dr. S.K. Kabra, Department of Pediatrics, Div. of Pediatric Pulmonology, AIIMS, New Delhi-110029.

E-mail : skkabra@hotmail.com pathogens are significant as far as severe lower respiratory tract infections are concerned, but the vast majority of acute respiratory infection are upper respiratory, and viral agents are the primary causal agents. In a hospital-based study on under-five children with acute respiratory infection, 38\% were upper respiratory tract infection. Viral etiological agent was detected in $16.6 \%$ and the viruses isolated were influenza A and B $(45.65 \%)$, parainfluenza 1 and $3(26.08 \%)$, adenovirus $(19.56 \%)$ and respiratory syncytial virus (6.52\%).

This bring forth the fact that, majority of respiratory infections on children are due to a viral etiology which do not need antibiotics for management and judicious use of antibiotics is most imperative in upper respiratory tract infections.

The principles of judicious use of antibiotics in upper respiratory tract infections outlined below are based on recent reviews.

\section{Common Cold}

Most children have 3-8 episodes of common cold per year. ${ }^{9-10}$ Common cold is a viral illness, the most common cause being rhinovirus; others being parainfluenza, respiratory syncytial, corona virus, adenovirus, echovirus, coxsackievirus and parainfluenza virus. ${ }^{11}$ Rhinovirus accounts for upto $60 \%$ of infections. ${ }^{12}$ The etiological agents vary with host age, and time of the year. Symptoms include nasal discharge, nasal obstruction, and throat irritation. Associated with this there usually is lowgrade fever, malaise, sneezing and nasal secretions can 


\section{N. Jain R. Lodha and S.K. Kabra}

become purulent. Symptoms are non-specific and not characteristic for any agent. The usual duration of symptoms is about 7 days.

Common cold in children resolves on its own and no specific therapy is indicated in majority of cases. Use of antibiotics does not change either the course or the outcome. ${ }^{6}$ On the contrary antibiotic use is potentially harmful, because it increases the risk of colonization with resistant organisms. This may result in subsequent bacterial infection being unresponsive to standard

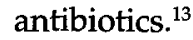

Common cold almost always has an excellent outcome with complete recovery, but at times complications can occur. These include acute otitis media with effusion, tonsillitis, sinusitis, and lower respiratory tract infection. ${ }^{11}$ Unless specific diagnostic criteria for the diagnosis of these complications are met, antibiotics should not be used for the signs and symptoms of cold alone. ${ }^{6}$ Studies have shown that use of antibiotics for the prevention of bacterial complication of common cold has no use. A meta-analysis of five randomized clinical trials of efficacy of antimicrobial treatment of cold to prevent lower respiratory infection found that it had no protective effect. It also did not shorten the duration of the upper respiratory illness. ${ }^{14}$

Recognizing the signs and symptoms of the usual course of common cold will help to prevent the overprescription of antibiotics. Unfamiliarity with them can lead to the mistaken diagnosis of secondary bacterial infection and the inappropriate use of antibiotics.

Mucopurulent nasal dicharge, which is thick, opaque at times greenish or yellowish frequently accompanies common cold. ${ }^{11}$ Antimicrobial treatment is not indicated for such a discharge unless it persists for more than 10-14 days. ${ }^{6}$

In a study on 142 children with mucopurulent nasopharyngitis, a comparison of cephalexin treatment with placebo was done. Five to six days after initiation of treatment, cephalexin and placebo groups did not differ in the presence of nasal discharge, incidence of complications, or parental opinion of benefit of medication. ${ }^{15}$

Cough occurs in $60-80 \%$ of common cold and does not suggest a bacterial etiology ${ }^{16}$ Duration of symptoms is usually 2-7 days. Patients usually improve within 10 days, but at times cough and nasal discharge can persist for 2 weeks or more. ${ }^{17}$

To summarize 1. Antibiotics should not be given for common cold. 2. Mucopurulent nasal discharge is feature of common cold and is alone not an indication of antibiotics unless it persists for more than 10-14 days.

\section{Sinusitis}

Sinusitis is defined as inflammation of the mucosal lining of one or more of the paranasal sinuses. ${ }^{18}$ It can occur from infection or non-infectious (allergic) causes. In children sinusitis almost always occurs as a complication of common cold. ${ }^{11}$ Even in uncomplicated viral upper respiratory infection, as occurs in common cold, there is inflammation and congestion of both the nasal and the sinus mucosa. These infections should, therefore, be considered as rhinosinusitis. ${ }^{19}$ This inflammation undergoes a spontaneous resolution in vast majority of cases. About 0.5 to 10 percent of upper respiratory infections are complicated by development of acute sinusitis. ${ }^{18}$ Since only a small percentage of children with symptoms of rhinosinusitis have a bacterial etiology, the use of appropriate diagnostic criteria is essential, as this will avoid the overuse of antibiotics. This is all the more important because viral rhinosinusitis is $20-200$ times more common than bacterial sinusitis. ${ }^{7}$

Sinusitis is classified as per the duration of symptoms into acute (upto 3 weeks), subacute (3-10 weeks), and chronic (>10 weeks).

Sinusitis is almost always precipitated when a viral upper respiratory infection produces mucosal inflammation leading to obstruction of the sinus ostia. This in turn leads to fluid trapping in the sinus cavities and the normal upper respiratory bacterial flora proliferates. The most common organisms causing sinusitis are $S$. pneumoniae, $H$. influenzae, $M$. catarrhalis, $S$. aureus and $S$. pyogenes. ${ }^{11}$ But it must be emphasized that majority of rhinosinusitis is viral and therefore, approximately $60 \%$ resolve spontaneously without antimicrobial therapy. ${ }^{20}$

The major problem in the diagnosis of sinusitis in children is to distinguish simple upper respiratory tract viral infections and allergic inflammation from bacterial infection of the sinuses. The signs and symptoms in older children can be classical i.e. sinus tenderness, tooth pain, headache, and high fever. However, in young children these classical sign and symptoms are almost never present. Persistence of signs and symptoms of upper respiratory infection i.e. rhinorrhea or cough for more than 10-14 days is a pointer towards sinusitis. The rhinorrhea mostly is purulent but can be serous or watery. Thus, the character of the discharge is not helpful in distinguishing infected sinus fluid, from uninfected fluid. ${ }^{21}$ Other indicators of sinusitis are the presence of severe signs and symptoms during an episode of upper respiratory infection. These include high fever $>39^{\circ} \mathrm{C}$, persistent fever, periorbital swelling and facial pain.

Diagnosis of sinusitis is clinical and rarely are investigations warranted. Radiographic evaluations are indicated when episodes of sinusitis are recurrent, when complications are suspected or when the diagnosis is not clear. ${ }^{7}$ The plain radiograph shows air fluid level, opacification, or mucosal thickening. ${ }^{18} \mathrm{CT}$ scan or MRI can be done for a more detailed examination and show the same findings. These features can also be present in viral rhinosinusitis and therefore, $\mathrm{X}$-ray should only be done when indicated. In a study evaluating CT findings in 31 adults during first 48-96 hours of uncomplicated viral respiratory illnesses, $90 \%$ had abnormality of the 


\section{Upper Respiratory Tract Infection}

maxillary sinus cavity. After two week these findings resolved in $79 \%$ even though none received any antibiotics. ${ }^{19}$ When the child is younger than six months of age, opacification of the ethmoid air cells is normal. When the child is under the age of one year, opacification or thickening of the mucosa of the maxillary sinusitis may be normal. ${ }^{11}$ This should be kept in mind when evaluating $X-$ ray findings.

The primary treatment of sinusitis is antibiotics once the diagnostic criteria have been met. Amoxycillin ( 40 $\mathrm{mg} / \mathrm{kg} /$ day) is successful for the initial treatment of acute uncomplicated sinusitis in most children. ${ }^{20}$ About $35 \%$ of nontypable $H$. influenzae and $85 \%$ of $M$. catarrhalis strains produce beta-lactamases and are resistant to amoxicillin. ${ }^{21}$

Therefore, if there is no response within 48 hours a beta- lactamase-stable antibiotic like amoxycillinclavulanate or cephalexin or cetactor should be considered. These drugs should be considered first line if sinusitis is recurrent, fails to improve, or is a severe infection with high fever and facial swelling. The duration of therapy should be minimum of 10 days. ${ }^{22}$

To summarize, 1 . Diagnosis of sinusitis should be made when symptoms of nonspecific upper respiratory infection are present for more than 10-14-days or there are severe upper respiratory signs and symptoms in the form. of facial pain, facial swelling and high fever. 2. Since radiological evidence of sinusitis may be seen even in common cold, radiographs should be interpreted with caution and done when indicated. 3. Antimicrobial for sinusitis should be narrow spectrum against the likely pathogen.

\section{Pharyngitis}

Pharyngitis is an inflammatory illness of the mucous membranes and underlying structures of the throat. The clinical diagnostic category includes tonsillitis, tonsillopharyngitis and nasopharyngitis. Pharyngitis is subdivided into two categories: illness with nasal symptomatology (nasopharyngitis) and illness without nasal involvement (pharyngitis or tonsillopharyngitis). Nasopharyngitis nearly always is a viral etiology, whereas pharyngitis without nasal signs has diverse etiologic possibilities including bacteria, viruses, fungi and other infectious agents. Adenoviruses are the most common cause of nasopharyngitis, other viruses being influenza, parainfluenza and enteroviruses. The bacterial agents causing pharyngitis include Streptococcus pyogenes, $H$. influenzae, $C$. diphtheriae, and $N$. meningitides. ${ }^{23}$

Group A streptococci accounts for approximately $15 \%$ of bacterial pharyngitis and about 1-2 patients out of 10 with sore throat. Infection in most of the others is attributable to viruses. ${ }^{24}$ Early diagnosis and treatment of Group A streptococcal pharyngitis is important, as antibiotics initiated with nine days of onset are effective in preventing acute rheumatic fever. ${ }^{25}$ But since most sore throats are viral in etiology antibiotic have to be given only when diagnosis of Group A streptococcal pharyngitis is certain. This will prevent the unnecessary overuse of antibiotics.

Symptoms of sore throat accompany pharyngitis but its presence alone should not be used for diagnosis as these complain can be present even in common cold. The objective evidence of inflammation in the form of erythema, exudate or ulceration in the pharynx is necessary for the diagnosis. But, these signs can also be present in some viral infections like adenovirus or enterovirus.

The classical features of Group A streptococcal pharyngitis include acute onset of pharyngeal pain, dysphagia and fever. Rhinorrhea, cough, hoarseness, conjunctivitis and diarrhea suggest a viral etiology. Presence of patchy exudate on the posterior pharynx, palatal petechie and enlarged and tender anterior cervical lymph nodes favour Group A streptococci pharyngitis. But in an individual child the clinical distinction between streptococcal pharyngitis and viral pharyngitis is unreliable. Therefore, in all cases of acute pharyngitis, streptococcal disease must be considered.

Diagnosis of Group A streptococcal pharyngitis should be based on throat swab culture, which is the standard for diagnosis. Antigen-detection tests alone can miss some cases; therefore the recommendation is to do a culture if antigen-detection test is negative in a child with suspected streptococcal pharyngitis. ${ }^{26}$ Furthermore, if antibiotics have been started in a child with suspected streptococcal pharyngitis and subsequently, the cultures are negative the treating physician should inform the parents to stop the antibiotic therapy. Some studies have shown that early initiation of antibiotic on clinical suspicion can result in decreased desirable antibody response, thus allowing reinfection with type specific organisms. ${ }^{27}$

Bacteria other than Group A streptococci are rare causes of pharyngitis. Moreover, sequelae such as acute rheumatic fever do not occur. Also, as already stated most pharyngitis in children is viral in etiology. Therefore, antibiotics should not be given to a child with pharyngitis in the absence of diagnosed Group A streptococcal pharyngitis or other bacterial infection.

The recommendation is to use 10-day course of penicillin for the treatment of Group A streptococcal pharyngitis. This is because it has a narrow spectrum of activity, low cost and high efficacy. Group A streptococcal resistance to macrolide antibiotics has been observed, whereas, resistance to beta-lactam antibiotics has not been detected to date. Resistance to macrolides like azithromycin or clarithromycin would be similar to that for erythromycin. A meta-analysis of 19 studies favoured cephalosporins over penicillin in terms of higher eradication of GABS from oropharynx..$^{28,29}$ These agents have a wide spectrum of activity against bacteria and thus encourage the emergence of resistance over a broad range of bacterial pathogens. Alternative treatments must be used in patients with penicillin allergy, compliance issues or penicillin treatment failure. Patients who do not 


\section{N. Jain R. Lodha and S.K. Kabra}

respond to initial treatment should be given an antimicrobial that is not inactivated by penicillinaseproducing organisms (e.g., amoxycillin-clavulanate potassium, cefaclor, cephalexin or a macrolides).

To summarize 1. Group A streptococcal pharyngitis should be diagnosed using laboratory tests with clinical and epidemiological findings. 2. Antibiotics should be given to a child with pharyngitis only when Group A streptococcal or other bacterial infection has been identified. 3. Penicillin is the drug of choice for Group A streptococcal pharyngitis.

\section{REFERENCES}

1. Manmohan, Bhargava SK. Acute respiratory infection. Indian Pediatr 1984; 211-213.

2. Chhabra P, Garg S, Mittal SK et al. Magnitude of acute respiratory infection in under five. Indian Pediatr 1993; 30 : 1315-1319.

3. Tambe MP, Sivaraman C, Chandrashekhar Y. Acute respiratory infection in Children-a survey in the rural community. Indian J Med Science 1999; 53 : 249-253.

4. Jain A, Pande PK, Mishra A et al. An Indian hospital study of viral causes of acute respiratory infections in children. J Med Microbiol 1991; 35 : 219-223.

5. Dowell SF, Schartz B. Resistant pneumococci: protecting patients through judicious antibiotic use. Am Fam Physician. 1997; 15 : 1647-1654.

6. Rosestein N, Phillips WR, Gerber MA et al. The common coldprinciples of judicious use of antimicrobial agents. Pediatrics 1998; 101( Suppl) : 181-184.

7. Dowell SF, Marcy M, Phillips WR et al. Principles of judicious use of antimicrobial agents for pediatric upper respiratory infections. Pediatrics 1998; 101 ( Suppl) : 163-165.

8. O'Brien KL, Dowell SF, Scharwtz B et al. Acute sinusitisprinciples of judicious use of antimicrobial agents. Pediatrics 1998; 101 (Suppl) : 174-177.

9. Schwartz B, Marcy SM, Phillips WR et al. PharyngitisPrinciples of judicious use of antimicrobial agents. Pediatrics 1998; 101 (Suppl) : 171-174

10. Wald ER, Dashefsky B, Byers $C$ et al. Frequency and severity of infections in daycare. J Pediatr 1988; 112 : 540-546.

11. Turner TB. The epidemiology, pathogenesis, and treatment of the common cold. Semin Pediatr Infect Dis 1995; 6 : 57-61.

12. MI Ashes. Infections of the upper respiratory tract. In Taussig LM, Landau LI, eds. Pediatric Respiratory Medicine. Mosby Inc Missouri 1999; 530-547.
13. Horn ME, Brain E, Gregg I et al. Respiratory viral infection in childhood. A survey in general practice, Rohanpton. 19671972. J Hyg 1975; 74 : 157-168.

14. Gadomski AM. Potential intervention for preventing pneumonia among young children : lack of effect of antibiotic treatment for upper resiratory infections. Pediatr Infect Dis I 1993; 12 : 115-120.

15. Todd JK, Todd N, Damato J et al. Bacteriology and treatment of purulent nasopharyngitis: a double blind, placebo controlled evaluation. Pediatr Infect Dis J 1984; 3 : 226-232.

16. Jackson GG, Dowling HF, Huldoon RL. Present concepts of the common cold. Am J Public Health 1962; 52 : 940-945.

17. Cloutier MM. The coughing child. Etiology and treatment of a common symptom. Postgrad Med 1983; $73: 169-175$.

18. Cherry JD, Newman A. Sinusitis. In Feigin RD, Cherry JD, eds. Textbook of Pediatric Infectious Diseases. 4th edn. WB Saunders, Philadelphia, PA. 1998; 183-192.

19. Gwaltney J, Phillips C, Miller R et al. Computed tomographic study of the common cold. N Eng J Med 1994; 330:25-30.

20. Giebink GS. Childhood sinusitis; pathophysiology, diagnosis and treatment. Pediatr Infect Dis I 1994; 13 : S55-S65.

21. Doern GV. Resistance among problem respiratory pathogens in pediatrics. Pediatr Infect Dis J 1995; $104: 462-463$.

22. Wald E. Sinusitis in children. N Eng J Med 1992; $326: 319-323$.

23. Cherry JD. Pharyngitis (Pharyngitis, tonsillitis, tonsillopharyngitis, and nasopharyngitis). In Feigin RD, Cherry JD, eds. TextBook of Pediatric Infectious Diseases. 4th edn. WB Saunders, Philadelphia, PA. 1998; 148-156.

24. Tanz RR, Shulman ST. Diagnosis and treatment of Group A streptococcal pharyngitis. Semin Pediatr Infect Dis 1995; 6 : 69 78 .

25. Rammelkamp $\mathrm{CH}$. Rheumatic heart disease-a challenge. Circulation 1958; $17: 842-851$

26. American Academy of Pediatrics. Group A streptococcal infections. In Peter G, ed. 1997 Red Book Report of The Committee on Infectious Diseases. 24th edn. Elk Grove Village, IL : American Academy of Pediatrics, 1997 : 83-94.

27. Pichichero ME, Disney FA, Green JL et al. Adverse and beneficial effects of immediate treatment of group A-betahemolytic streptococcal pharyngitis with penicillin. Pediatr Infect Dis 1987; $6: 635-643$.

28. Pichichero ME, Margolis PA. Comparison of cephalosporin and penicillins in the treatment of group A beta-hemolytic streptococcal pharyngitis: a metaanylysis supporting the concept of microbial copathogenicity. Pediatr Infect Dis J 1991; $10: 275-281$.

29. Jacobs RF. Judicious use of antibiotics for common pediatric respiratory infections. Pediatr Infect Dis J 2000; 19 : 938-943. 